who had not previously shown impairment of ventilation, 23 were unchanged, but 2 had deteriorated. Of the 19 who did have reduced ventilatory capacity 8 had improved to normal levels, 4 had improved but still had some impairment, 3 were worse, and 4 were unchanged. A further nine workers were retested who were not sensitized but had impaired ventilation in May. Seven had improved, but five of these were still below normal level; the remaining two were worse.

Two points of interest emerged at this stage: (1) the one subject whose skin test reaction to enzyme extracts had converted to positive did so in a period with only six days' exposure to the $B$. subtilis derivative, and (2) in this population the impairment of lung function could be quite severe, and in several cases had not reverted to normal during 11 weeks without contact with $B$. subtilis derivative.
Acknowledgements are due to Dr. T. A. Lloyd Davies, Senior Medical Inspector of Factories, for his guidance and support and for permission to publish; to Professor J. Pepys and the department of clinical immunology, Institute of Diseases of the Chest, for advice, the supply of skin test materials, and serological testing; also to the members of the Factory Inspectorate Medical Branch who helped to carry out the survey (Dr. J. G. S. West, Dr. R. Whitelaw, and Dr. R. Morley).

Requests for reprints should be addressed to Dr. M. Greenberg.

\section{REFERENCES}

College of General Practitioners (1961). British Medical fournal, 2, 973. Flindt, M. L. H. (1969). Lancet, 1, 1177.

Lloyd Davies, T. A. (1970). Report of the Survey of Respiratory Disease in Foundrymen. In press.

Pepys, J. (1969). Hypersensitivity Diseases of the Lungs due to Fungi and Organic Dusts. Basel, Karger.

Pepys, J., Hargreave, F. E., Longbottom, J. L., and Faux, J. (1969). Lancet, 1, 1181

\title{
Method for Measuring Cerebral Dysfunction in Patients with Liver Disease
}

\author{
R. ZEEGEN, ${ }^{*}$ M.B., D.OBST.R.C.O.G.; J. E. DRINKWATER, † B.SC., DIP.PSYCH., A.B.PS.S.; A. M. DAWSON,
}

\begin{abstract}
Cummary: Objective tests of cerebral function were undertaken in 64 outpatients after portal decompression for intrahepatic portal hypertension. These involved scoring the errors in constructing a five-pointed star, and a simple modification of the Reitan trail making test. The first test was found to be less discriminating than the second. There was some correlation between the results and the presence or absence of overt encephalopathy, which-though generally mild-was present in $\mathbf{3 0}$ $(47 \%)$ of the 64 patients.
\end{abstract}

\section{Introduction}

Neuropsychiatric changes may frequently complicate chronic liver disease, especially after portal decompression. Hitherto means of accurate assessment of these changes has been lacking and usually clinical impression has been relied on, when the incidence of portal systemic encephalopathy following portal decompression has been variously estimated as from $13 \%$ (Renwick et al., 1969) to 52\% (Resnick et al., 1969). Grace et al. (1966) calculated a overall incidence of $19 \%$ in 733 patients collected from 13 groups. As mental changes probably occur more often that overt neurological signs, this present study was undertaken, by means of a test designed to demonstrate brain damage, to assess the incidence of cerebral dysfunction in such patients.

This was done, firstly, by devising a scoring system for errors made in drawing a five-pointed star, indicating the constructional apraxia which occurs in hepatic encephalopathy (Davidson and Summerskill, 1956), and, secondly, by applying a modified version of the Reitan trail making test (Reitan, $1955,1958)$. This is a validated test for distinguishing between the presence or absence of organic brain damage; limitations of the test, particularly with psychotic patients, have been fully reviewed (Brown et al., 1958; Goldstein and Neuringer, 1966). The incidence of impaired mental function assessed in these ways could then be compared and contrasted with the incidence of clinically overt encephalopathy.

\footnotetext{
* Research Fellow.

† Principal Psychologist.

$\neq$ Physician.

St. Bartholomew's Hospital, London E.C.1.
}

\section{Patients and Methods}

Control Group.-This comprised 25 patients selected from routine outpatient clinics who could reasonably be assumed to have no organic mental damage and whose clinical examination had disclosed no abnormality. There were 10 men and 15 women in the group, aged from 23 to 73 years. Diagnoses included peptic ulcer, back pain, anxiety state, ulcerative colitis, irritable bowel, and ankle oedema of uncertain aetiology.

Patients.-Sixty-four patients were studied by testing those attending a routine follow-up clinic from March 1968 to October 1969. All had undergone portal decompression for intrahepatic portal hypertension from three months to 19 years previously; this represented a follow-up of over $90 \%$ of the available patients. There were $51(80 \%)$ with hepatic cirrhosis of mixed aetiology, $12(19 \%)$ with "minor" liver changes (Zeegen et al., 1970b), and one with congenital hepatic fibrosis (Kerr et al., 1961). The group comprised 37 men and 27 women aged 14 to 75 years. There was no significant difference in age between the patients and the controls (see Table).

Ages and Incidence of Abnormal Star Error Score and Combined $A$ and $B$ Times of the Reitan Trail Making Test for Control Subjects and Patients

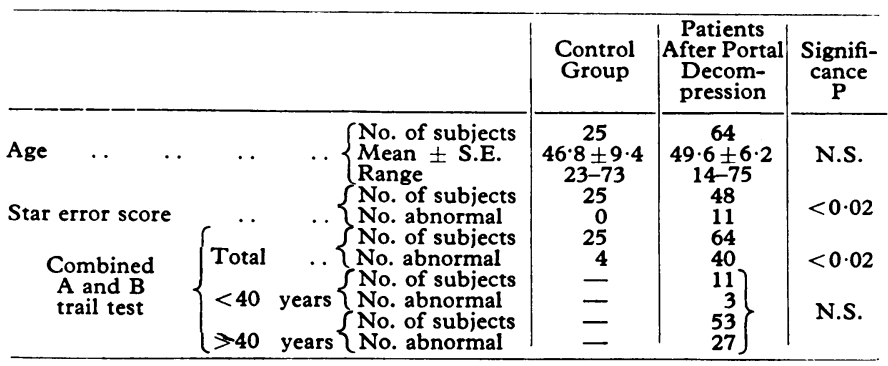

\section{Tests Employed}

Error. Score for Construction of a Five-pointed Star.-Each patient was asked to copy the star accurately. The scoring method was as follows: (A) score one for each sharp star point to a maximum of five, deduct one from the total for each sharp star point in excess of five; (B) score one 
for each sharp inner angle to a maximum of five, deduct one from the total for each angle in excess of five; (C) score one for each sharp inner angle greater than 90 degrees but less than 180 degrees (measured if necessary) to a maximum of five; sharp angles enclosed by curved lines considered as the angle between straight lines of best fit; (D) score one for each star point of roughly equal overall length and width to a maximum of five (count only star points with each side of about equal length), deduct one for each star point in excess of five. The sum of the scores from A, B, C, and D was subtracted from 20 to give the error-score. Scoring was reproducible when two observers were tested against one another for each group (Pearson product moment correlation coefficient: $r=0.85$ and 0.97 for control and outpatients respectively), and thus analyses were carried out with data from one observer only.

Modified Reitan Trail Making Test.-The test comprised two tasks. After a standard explanation the patient joined up in the correct sequence a series of encircled numbers (Fig. 1, test $\mathrm{A}$ ) and then numbers and letters alternately (Fig. 2, test B). The efforts were scored according to the time taken for completion, including mistakes, which were dealt with by instructing the patient to return to the preceding correct number or letter and then carry on. To avoid practice effects in sequential testing, three variations in addition to the standard tests were obtained by rotating the standard cards through 90-degree positions and reprinting with appropriate reorientation of numbers and letters. The test cards were then selected randomly. No convincing practice effect was found in this group, nor in a small group of inpatients with chronic encephalopathy reported elsewhere (Zeegen et al. 1970a), where the test was used sequentially. Reitan considered the most discriminating readings were the $B$ trail test times, and the combined $\mathbf{A}$ and $\mathbf{B}$ times. Patients unable to complete test $\mathbf{B}$ were very severely impaired.

The tests were explained to the controls and patients as part of research project; no individual refused to co-operate.
(15)

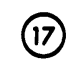

(20)

(18)

(5)

(4)

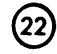

(13)

(44)<smiles>[18OH]</smiles>

()

(7)

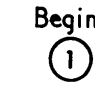

(24)

(8) (10) (2)

(9)

(12)
(13) (8)
(1)

(2)

$\stackrel{\text { m }}{\frac{1}{2}}$

(B)

(4)

(3)<smiles>O</smiles>

Begin

(5)

(H)

(c)

(12)

G)

(A)

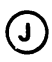

(E)

(K)

FIG. 2.-Part $B$ of the Reitan trail making test. The patient was instructed to join up the numbers and letters alternatively in the correct sequence--that is, 1 to $A$ to 2 to $B$ to 3 to $C$, etc. The effort was timed to completion.

Where more than one reading was obtained in a patient, the first set of complete readings were used for purposes of comparison.

\section{Clinical Neuropsychiatric Grading}

The following simple grading system was used each time the foregoing tests were applied : $0=$ no detectable abnormality, $\mathrm{I}=$ mild mental changes and brisk reflexes (mild encephalopathy), II = disorientation, of ten confusion (severe encephalopathy), and III = coma.

\section{Biochemistry}

In addition to regular routine liver functions tests, venous plasma ammonia levels were measured by the ion-exchange method of Fenton and Williams (1968) on each attendance at the Portal Hypertension Clinic.

\section{Results \\ Clinical Neuropsychiatric Grade}

Thirty-four patients (53\%) had no detectable abnormality and were therefore grade 0 ; of the remaining 30 patients 27 had a mild degree of encephalopathy (grade I), and three were grade II. Somewhat surprisingly there was no difference in age between patients with and without clinical encephalopathy, the mean age of both groups being 49.6 years.

\section{Psychometric Tests}

Star Error Score.-The error score of the controls ranged from 0 to 9 , and it was shown statistically that a score of above 9 was abnormal for this distribution. This test was less sensitive than the clinical scale in detecting mental dysfunc-

FIG. 1.-Part A of the Reitan trail making test. After a standard explanation the patient was instructed to join up the numbers in sequence as rapidly as possible. The effort was timed to completion. 
tion, as $11(23 \%)$ of the 48 patients tested gave abnormal star error scores, as compared with $24(50 \%)$ with clinical encephalopathy. Of the 11 patients with abnormal scores all but three had clinical encephalopathy.

Reitan Trail Making Test.- From Reitan's series we took a combined A and B test time of over 130 seconds as being abnormal and compatible with the presence of brain damage. Four (16\%) of the 25 controls gave abnormal A and B times, but it should be pointed out that three of these times were less than 140 seconds and only one represented severe impairment (Fig. 3). The incidence of cerebal dysfunction in the patients after portal decompression was surprisingly high, and greater than was clinically obvious. In fact, $40(62.5 \%)$ of the 64 gave combined times in the brain damage range, including those seven who were unable to complete part B. The patients with clinical encephalopathy were the most severly impaired, for only $3(10 \%)$ out of 30 with grade I or II encephalopathy gave normal combined A and B times, in contrast with $21(62 \%)$ of 34 patients with no overt encephalopathy $(\mathrm{P}<0.01)$. The overall scatter of readings of the combined times of the two groups is shown in Fig. 3. Statistical analysis of these times was made difficult because of the seven severely impaired patients who were unable to complete test $B$. The times however were still significantly different when compared with the times of those patients who com-, pleted the test (Fig. 3). There was a tendency for older

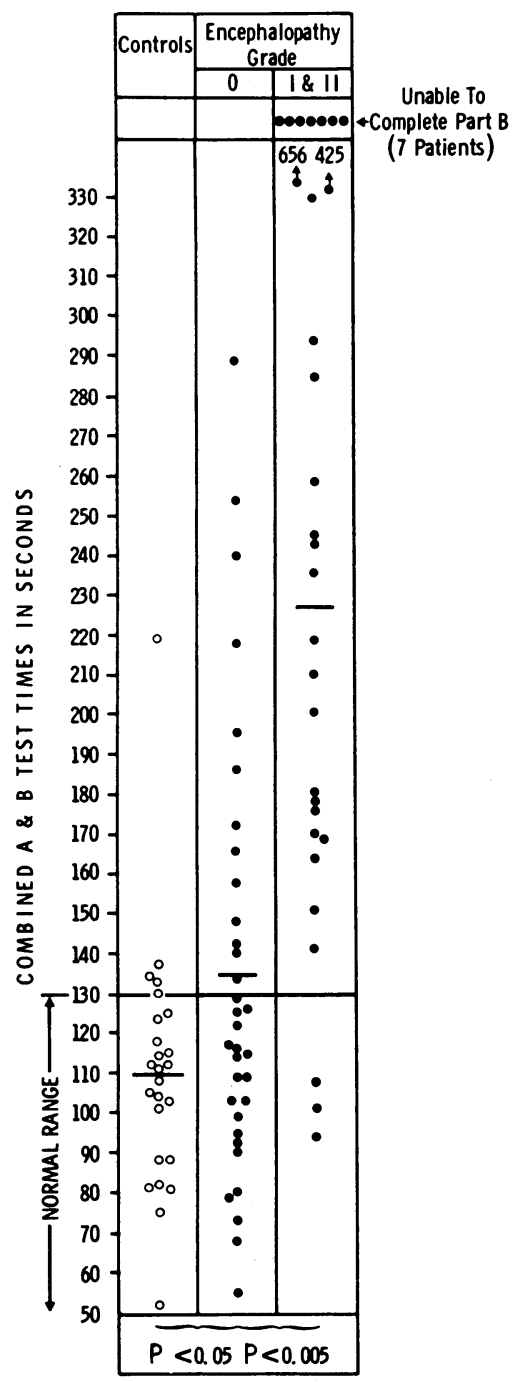

Fig. 3.-Combined A and B test times of the Reitan trail making test for controls and patients following portal decompression with and without clinical encephalopathy. Means for each group are shown as horizontal clinical encephalopathy. Means for each group are shown as horizontal
bars (110 seconds, 134.1 seconds, and 227.6 seconds respectively). Differences between these are highly significant even excluding the seven encephalopathic patients unable to complete part $B$. patients, after portal decompression, to have a greater impairment in ability to perform the tests, though this did not quite reach statistical significance. Furthermore, there was no correlation between trail test performance and duration after operation.

\section{Plasma Ammonia Levels}

There was no correlation between the venous ammonia levels and either the trail making test results or the presence or absence of clinical encephalopathy. This was not surprising, as the samples were non-fasting; but during sequential studies on seven inpatients with chronic hepatic encephalopathy reported elsewhere (Zeegen et al., 1970a), where fasting arterial samples were taken, the values often paralleled the clinical state and the trail test performance.

\section{Discussion}

So far as we are aware this is the first occasion that objective psychometric tests have been applied to quantitate the mental changes in patients after porta-caval anastomosis. Of the two methods used, the technique devised for scoring the five-pointed star, though giving reproducible results, was the less satisfactory. The scoring system was complex and the discriminative function of the method was poor as it underestimated the incidence of mental changes as determined clinically and by the trail making test.

Trail making test results in the present control group were closely comparable to those reported by Reitan (1958). When combined A and B test times were considered $4(16 \%)$ of the control subjects were misclassified as abnormal, as were 14 (16.7\%) of 84 controls in Reitan's series. Conversely, the test misclassified as normal only $3(10 \%)$ patients with overt encephalopathy, as compared with normal times given by 28 (14\%) of 200 of Reitan's patients with organic brain damage. The abnormal trail test times in those patients following portal decompression probably suggest encephalopathy: firstly because the incidence of abnormalities was much greater in those patients with overt encephalopathy (Fig. 3); and, secondly, because trail making test performance tended to change pari passu with clinical state during remission of encephalopatby induced by inpatient treatment with lactulose (Zeegen et al., 1970a) even when they were initially unable to complete the test. This would also suggest that, in this group of patients at least, impairment of trail making test ability may not indicate permanent organic brain damage, though patients dying with hepatic encephalopathy show cortical neuronal degeneration and abnormal astrocytes (Adams and Foley, 1959).

The trail test has shown that many patients in this series had cerebral dysfunction; for abnormal results were obtained in virtually two-thirds of the 64 outpatients. Furthermore, this group represented a follow-up of at least $90 \%$ of the patients surviving portal decompression. This high incidence was not expected, as most patients were leading apparently normal lives and several were working. In addition the incidence of clinical encephalopathy (30 (47\%) out of 64 patients) was greater than that quoted in other series (Grace et al., 1966; McDermott et al., 1968). All except 3 of the 30 patients, however, had mild encephalopathy, and when the incidence of severe encephalopathy requiring admission to hospital was considered the figure was $18.4 \%$ of a group of 251 patients after portal decompression (Zeegen et al., 1970a), some of whom were included in the present series. This finding was then comparable with that of Grace et al., (1966) and of McDermott et al. (1968). Such a large proportion of patients with cerebral dysfunction and clinical encephalopathy-albeit mild-may have been in part due to self-selection, for patients attended more frequently when they were unwell. The impression, however, was that most patients attended only for routine appointments, and where 
multiple values were obtained from several attendances only the first complete set were analysed.

It has usually been suggested that portal systemic encephalopathy is more likely to occur in older patients (Rousselot et al., 1963; McDermott et al., 1968; Panke et al., 1968). This was not so in the present series, for though the incidence of cerebral dysfunction tended to be greater in patients aged 40 and over there was no significant age distribution to the overall incidence of clinical encephalopathy.

The Reitan trail making test has afforded a useful tool for the objective assessment of cerebral dysfunction after portal decompression and has also been used sequentially to measure response to treatment of encephalopathy. At the moment the sociological significance of these alterations in cerebral function is uncertain. They may well be important in patients whose occupation demands a moderate intellectual capacity, and to clarify this point further work is being undertaken.

This group of patients had no other cause for cerebral dysfunction, such as alcoholism, and the use of these tests in assessing cerebral changes in patients who have undergone portal decompression may be limited in alcoholic patients.

We would like to thank Mr. Alan Hunt for allowing us to study patients under his care, and the Board of Governors of St. Bartholomew's Hospital for financial support.

\section{REFERENCES}

Adams, R. D., and Foley, J. M. (1953). Research Publications. Association for Research in Nervous and Mental Disease, 32, 198.

Brown, E. C., Casey, A., Fisch, R. I., and Neuringer, C. (1958). fournal of Consulting Psychology, 22, 469.

Davidson, E. A., and Summerskill, W. H. J. (1956). Postgraduate Medical fournal, 32, 487.

Fenton, J. C. B., and Williams, A. H. (1968). fournal of Clinical Pathology, 21, 14.

Goldstein, G., and Neuringer, C. (1966). Perceptual and Motor Skills, $22,347$.

Grace, N. D., Muench, H., and Chalmers, T. C. (1966). Gastroenterology, $50,684$.

Kerr, D. N. S., Harrison, C. V., Sherlock, S., and Walker, R. M. (1961). Quarterly fournal of Medicine, 30, 91 .

McDermott, W. V., Barnes, B. A., Nardi, G. L. and Ackroyd, F. W. (1968). Surgery, Gynecology and Obstetrics, 126, 585.

Panke, W. F., Rousselot, L. M., and Burchell, A. R. (1968). Annals of Surgery, 168, 957 .

Reitan, R. M. (1955). Fournal of Consulting Psychology, 19, 393.

Reitan, R. M. (1958). Perceptual and Motor Skills, 8, 271.

Resnick, R. H. et al. (1969). Annals of Internal Medicine, 70, 675

Renwick, S. B., Loewenthal, J., and Mills, F. H. (1969). Medical fournal of Australia, 1, 755 .

Rousselot, L. M. Panke, W. F., Bono, R. F., and Moreno, A. H. (1963). American fournal of Medicine, 34, 297.

Zeegen, R., Drinkwater, J. E., Fenton, J. C. B., Vince, A., and Dawson, A. M.' (1970a). Quarterly fournal of Medicine. In press.

Zeegen, R., Stansfeld, A. G., Dawson, A. M., and Hunt, A. H. (1970b). Gut. In press.

\title{
Placental Localization by Isotope Scanning with ${ }^{113 \mathrm{~m} I n}$. Results in 200 Patients
}

\author{
F. W. WRIGHT,* B.M., M.R.C.P., F.F.R.
}

\begin{abstract}
Summary: Placental scanning was carried out in 200 $\checkmark$ patients over a period of 22 months with ${ }^{113 m} \mathrm{In}$. It was found to be a reliable method of demonstrating the placenta, with a smaller radiation dosage than by $x$-ray methods.
\end{abstract}

\section{Introduction}

Placental localization is now being carried out more frequently either to confirm placenta praevia or, if this is excluded, to allow the expectant mothers who have been admitted to hospital to return home for social reasons or to free hospital beds for others. If reliance is to be placed on the localization then it must be accurate and should be convenient and without risk to mother and fetus.

\section{Methods}

$X$-ray Methods.-Soft-tissue radiography may demonstrate the placenta by visualizing fine calcification within it in $30-50 \%$ of patients with a whole-body radiation dose to the fetus of 100 to 2,000 mrads. Erect lateral pelvimetry will usually exclude a major degree of placenta praevia if the fetal head is engaged in the pelvis (Reid, 1949, 1951; Vickers, 1965). This method can give rise to false-negative and falsepositive diagnoses, and the engaged head may not exclude a major degree of placenta praevia (Macafee, 1956). The most reliable $x$-ray methods are angiography and amniography. In the former a catheter is inserted from the femoral artery up to the bifurcation of the aorta, and after injection of contrast medium one or two radiographs are taken of the whole abdomen or pelvis (Fernström, 1955, Brink, 1960; Sutton, 1966; and Herlinger, 1968). In amniography the contrast medium is injected into the amniotic sac.

\footnotetext{
* Consultant Radiologist, United Oxford Hospitals (Churchill Hospital, Oxford).

† SELO = Societa Elettronica Lombarda; Milan, Italy. (U.K. agents : Tracerlab (G.B.) Ltd.)
}

$\ddagger$ The Radiochemical Centre, Amersham.
Thermography and Ultrasound.-I have not found thermography to be of value. Unless the placenta is anterior there seems little chance of its detection by this method. Doptone examinations have confirmed the position of the placenta in some patients. Compound B scans are capable of showing the placenta even in the first trimester and are considered by Donald (1969) and Secker Walker et al. (1969) to be the method of choice. More recently, Cryer et al. (1970) stated, "at present, it would appear that neither ultrasonics nor thermography is a serious competitor to radio-isotope and radiographic methods," and also suggested that detailed follow-up studies should be made in order to detect any possible harmful effects of the diagnostic use of ultrasound.

Isotope Methods.-All isotope methods depend on labelling the maternal blood and detecting the placental blood pool. Browne (1951) used ${ }^{24} \mathrm{Na}$, and others have used ${ }^{131} \mathrm{I}$ and ${ }^{99 \mathrm{~m}} \mathrm{Tc}$ serum albumin with physical half-lives of 8 days and 6 hours respectively, and have used thyroid-blocking agents to help prevent uptake of isotope in maternal and fetal thyroids. Inhaled gases, such as ${ }^{11} \mathrm{CO}$, with physical half-life of 20 minutes (Jacoby and Arnot, 1969), may also be used to label the blood, if there is a nearby cyclotron to produce the isotope. The present isotope of choice is ${ }^{113 \mathrm{~m}} \mathrm{In}$ (physical half-life 1.7 hours), which is derived from a ${ }^{113} \mathrm{Sn}$ generator column. The gamma energy is $393 \mathrm{KeV}$, which means that little radiation is absorbed or scattered in the tissues as may occur using ${ }^{99 m} \mathrm{Tc}(140 \mathrm{KeV})$ with a posteriorly situated placenta.

Technique.-The column is eluted with $4 \mathrm{ml}$. of $\mathrm{N} / 20$ $\mathrm{HCl}, 0.5 \mathrm{ml}$. of $20 \%$ gelatin is added, and the mixture autoclaved. Then $250-500 \mu \mathrm{Ci}$ is injected intravenously at about pH 1.6-a modification of the original method of Stern et al. (1967) (see also Wagner, 1968). Scans are made with the patient first supine, after she has emptied her bladder, and subsequently lateral, if the placenta is not fundal or is poorly seen. A dual 5-in. (12.5-cm.) SELOt scanner is used, summing the outputs of the detectors (above and below the patient) in the 